\title{
MORTALIDAD POR EPIDEMIAS Y ENDEMIA SEGÚN CAUSAS Y CONDICIONES SANITARIAS A MEDIADOS DEL SIGLO XIX EN LIMA, PERÚ
}

\author{
Carlota Casalino ${ }^{1,2, a}$
}

RESUMEN

A mediados del siglo XIX en Lima, Perú, las personas morían por diversas causas. Pero eran las epidemias las que generaban consternación y preocupación en la población y en las autoridades. Sin embargo, el número mayor de muertes fue por endemias debido a las pésimas condiciones sanitarias de la ciudad. Pero como estas eran muertes cotidianas pasaron desapercibidas.

Palabras clave: Epidemia; Endemia; Lima (fuente: DeCS BIREME).

\section{EPIDEMIC AND ENDEMIC MORTALITY ACCORDING TO CAUSES AND SANITARY CONDITIONS IN MID-NINETEENTH CENTURY LIMA, PERU}

\begin{abstract}
In mid-19th century Lima, Peru, death had various causes. Nonetheless, epidemics raised greater concern among the population and authorities. The highest number of deaths was due to endemic diseases caused by poor sanitary conditions. However, as these were accepted as routine deaths, they were ignored.
\end{abstract}

Key words: Epidemic; Endemic; Lima (source: MeSH NLM).

\section{INTRODUCCIÓN}

En la ciudad de Lima entre 1840 y 1860 las personas morían por distintos factores. $Y$ fue durante las epidemias cuando la población y las autoridades tomaron medidas. Sin embargo, en este artículo se mostrará que más gente falleció por endemias debido a las pésimas condiciones sanitarias de la ciudad; solo que las víctimas, al ser parte de todos los días, no llamaron tanto la atención. Dado que este artículo se basa en el análisis de las epidemias y la endemia, consideramos pertinente definir ambos términos. Para la OMS la epidemia es Aquella enfermedad contagiosa o no, que agrede o embiste a una gran cantidad de individuos pertenecientes a un mismo lugar y durante un periodo definido. En el caso de la endemia: Son enfermedades que se mantienen dentro de los límites inferiores del Reglamento Sanitario Internacional (1).
El periodo estudiado se ubica en el paradigma precientífico y, por lo tanto, se atendían los síntomas y no las causas de las enfermedades; es un periodo anterior a Pasteur, previo a la microbiología y la bacteriología. Al paradigma de los humores y miasmas de la antigüedad se añadió la observación anatómica y la experimentación del mundo moderno, pero faltaba aún identificar los vectores y el mundo microbiano. También hubo debates entre los contagionistas y los anticontagionistas.

Autoridades y médicos de entonces retomaron el asunto de la higiene pública. Este concepto fue introducido por los borbones en el periodo tardo colonial. Luego, a mediados del siglo XIX, Manuel A. Fuentes propuso que el Estado dicte medidas a favor la higiene pública para lograr una población saludable. Como parte del contexto, es preciso mencionar que el Perú incrementó significativamente sus ingresos por el boom del guano, por lo que el Estado pudo

\footnotetext{
Universidad Peruana de Ciencias Aplicadas. Lima, Perú.

Facultad de Ciencias Sociales, Universidad Nacional Mayor de San Marcos. Lima, Perú.

a Historiadora. Doctora en Ciencias Sociales.

Recibido: 07/11/2016 Aprobado: 24/05/2017 En línea: 29/09/2017
}

Citar como: Casalino C. Mortalidad por epidemias y endemia según causas y condiciones sanitarias a mediados del siglo XIX en Lima, Perú. Rev Peru Med Exp Salud Publica. 2017;34(3):564-8.doi: 10.17843/rpmesp.2017.343.2486 
crecer y hubo relativa estabilidad política. Esto permitió mejorar las condiciones de la ciudad.

La ciudad de Lima tenía registrados 60 médicos en 1860. Además, fue un periodo muy importante, porque se reformó la Escuela de Medicina y la Escuela de Cirugía. Periodo en el cual destacó Cayetano Heredia ${ }^{(2)}$.

A continuación, presentamos un acápite sobre las causas de muerte por enfermedades en tiempos "normales" y en tiempos "de epidemia" con información tomada del libro de registro del Cementerio General ${ }^{(3)}$. Luego, en otro acápite, vincularemos esa información con las condiciones de la ciudad y con la higiene pública.

\section{MUERTE EN LIMA A MEDIADOS DEL SIGLO XIX}

Como en toda sociedad, las causas por las cuales mueren sus habitantes son muy diversas. Si las agrupamos, podemos facilitar el análisis. Resultado de ello, encontramos causas físicas, degenerativas, enfermedades infecciosas, enfermedades no infecciosas y epidemias.

Del periodo 1840-1860 hemos elegido algunos años. Primero seleccionamos los lustros: 1840, 1845, 1850,
1855 y 1860. Luego identificamos los años "especiales", cuando las cifras de muertos se elevan por encima del promedio: 1854, 1856, 1858 y 1859.

El primer grupo fue denominado "años normales". En 1840 se asentó un total de 2339 casos; en 1845 fueron 3251; en 1850 se apuntaron 3594; en 1855 fueron 4838, y en 1860 hubo 3459 .

El segundo grupo fue denominado "años de epidemia", debido a que, en esos años, la presencia de fiebre amarilla explica el incremento de fallecidos en 1854 y 1856; y la presencia de viruela en 1858 y 1859 . Además, 1855 y 1860 tienen casos de fiebre amarilla y viruela, respectivamente.

\section{MUERTE EN “TIEMPOS NORMALES”}

En la Tabla 1 podemos apreciar que entre las principales causas de muerte por enfermedades infecciosas están costado, disentería, fiebres y tisis. Nótese que se mantuvo la denominación utilizada por la fuente y que se agruparon algunas al referirse a lo mismo.

Observamos que más del $50 \%$ de causas de muerte se debieron a enfermedades infecciosas. Destacando en 1840 y 1845 la disentería; y en 1850 y 1860 las "fiebres".

Tabla 1. Causas de muerte en Lima en "tiempos normales" (porcentajes)

\begin{tabular}{|c|c|c|c|c|c|}
\hline Causa & 1840 & 1845 & 1850 & 1855 & 1860 \\
\hline Aire & 0,54 & 0,27 & 0,65 & 0,15 & 0,26 \\
\hline Angina & 0,82 & 0,11 & 0,21 & 0,73 & 1,05 \\
\hline Asma, ahogos & 0,16 & 0,15 & 0,14 & 0,06 & 0,11 \\
\hline Cólera, Colerín y cólera morbus & 0,21 & 0,19 & 0,32 & 0,12 & 0,86 \\
\hline Colitis & --- & --- & 0,39 & 0,06 & --- \\
\hline Costado, pleuresía & 9,53 & 4,30 & 4,27 & 2,36 & 2,17 \\
\hline Dientes, Colmillos, dentición, dientes & 1,47 & 0,63 & 0,50 & 0,47 & 1,27 \\
\hline Disentería, bicho & 20,00 & 23,98 & 11,22 & 13,34 & 11 \\
\hline Empacho & 0,49 & 0,79 & 0,50 & 0,19 & 0,37 \\
\hline Fiebres & 11,17 & 21,55 & 19,80 & 23,24 & 14,61 \\
\hline Fiebre amarilla & --- & --- & --- & 0,67 & 0,07 \\
\hline Fístulas & 0,21 & 0,07 & 0,18 & 0,15 & 0,18 \\
\hline Gangrena & 0,54 & 0,07 & 0,08 & 0,31 & 0,60 \\
\hline Hidropesía & 3,83 & 2,43 & 1,55 & 1,53 & 1,91 \\
\hline Inflamación & 0,32 & 0,75 & 0,07 & --- & 0,26 \\
\hline Mal de 7 días & 0,76 & 0,91 & 0,07 & 0,03 & 0,48 \\
\hline Mal de orina & 0,60 & 0,35 & 0,32 & --- & 0,33 \\
\hline Mal de parto, sobreparto y parto & 0,10 & --- & --- & 0,41 & 0,37 \\
\hline Pecho, pecho dañado & 0,82 & 0,19 & 0,18 & 0,22 & 0,22 \\
\hline Pulmonía, pulmones & 1,20 & 0,79 & 1,30 & 2,20 & 5,48 \\
\hline Tabardillo, fiebres pútridas & 4,00 & 2,31 & 1,04 & 1,24 & 1,08 \\
\hline Tisis, Sangre de boca y tubérculos & 11,28 & 11,39 & 12,12 & 11,30 & 15,62 \\
\hline Ulcerado, úlceras & 0,21 & 0,47 & 0,07 & 0,25 & 0,30 \\
\hline Viruelas & 0,16 & 1,19 & 0,97 & 0,03 & 2,32 \\
\hline Otros & 31,58 & 27,11 & 44,03 & 40,94 & 39,08 \\
\hline
\end{tabular}

Tomado de Carlota Casalino, La Muerte en Lima en el s. XIX, p. 270. 
La disentería fue causa de muerte durante todo el año, se la relaciona con malas condiciones de higiene. El agente patógeno es la Entamoeba histolytica, ameba que vive frecuentemente en el colon sin hacer daño, pero bajo condiciones de estrés, bajas resistencias, o un cuadro particularmente patógeno, invaden las paredes intestinales y causan abscesos. La infección tiene una ruta fecal-oral y la infección directa puede ocurrir en circunstancias de extremo hacinamiento. La infección indirecta más común es la contaminación fecal de la comida y el agua, cuando hay alta contaminación de cañerías y grifos ${ }^{(4)}$.

En 1840, los casos por disentería fueron 364, los más altos fueron febrero y diciembre con 44 y 41 casos respectivamente. En 1845, los casos se incrementaron a 602 , siendo julio más alto con 92. Los demás años, 1850 , 1855 y 1860 tienen 310, 418 y 193 casos respectivamente, de marzo a junio la frecuencia fue ligeramente más alta que el resto del año.

Respecto a las "fiebres", que aludían al síntoma de una infección y solían ser clasificadas por periodo de acceso (quotidianas, tercianas o quaternarias); intensidad (benignas, graves y perniciosas); carácter (inflamatorias y pútridas); causas ocasionales o remotas, locación en el cuerpo (cephalicas, cardiálgicas, hepáticas, etc.); efectos (depuratorias y corruptivas), y de orden y proporción de su actividad (periódicas o erráticas). Las dos primeras corresponden a la malaria que en América en general, y en Lima en particular, se volvió endémica con la llegada de españoles a partir del siglo XV, ${ }^{(5)}$ al igual que el sarampión o la viruela ${ }^{(6)}$

Las "fiebres" fueron causas de muerte en todo el año, en algunos meses las cifras se incrementaban. En 1840 la mayor frecuencia fue marzo, abril y mayo con 32, 28 y 32 casos respectivamente. En 1845 en esos mismos meses hubo 81,63 y 57 casos. Pero en 1850 y en 1855 los meses fueron abril, mayo y junio; en 1860 el incremento fue en marzo, abril y mayo.

La disentería y las "fiebres" eran padecimientos que provocaban la muerte de los habitantes de Lima a lo largo de los años estudiados y en ese entonces eran endémicas.

\section{MUERTE EN “TIEMPOS DE EPIDEMIA”}

Identificamos cuatro años de epidemias e incluimos dos años "normales", porque las causas de muerte por fiebre amarilla en 1855 y el de viruela en 1860 si bien son más bajos que el año precedente, tienen cifras por encima de "lo normal". En la Tabla 2 se puede apreciar que las muertes por fiebre amarilla corresponden a 1854 y 1856 y los de viruela a 1858 y 1859 . No consideramos la gran epidemia de 1867-1869, analizada por Rottenbacher de Rojas en un sugerente trabajo donde estudia los efectos que tuvo en la sociedad $^{(7)}$

Tabla 2. Causas de muerte en Lima en "tiempos de epidemia".

\begin{tabular}{|c|c|c|c|c|c|c|}
\hline \multirow{2}{*}{ Causas } & \multicolumn{6}{|c|}{ Número de muertes } \\
\hline & 1854 & 1855 & 1856 & 1858 & 1859 & 1860 \\
\hline Aire & 5 & 5 & 6 & 5 & 4 & 7 \\
\hline Angina & 6 & 23 & 10 & 41 & 35 & 28 \\
\hline Asma & 7 & 2 & -- & -- & 5 & 2 \\
\hline Cólera, colerín, & 5 & 4 & 12 & 7 & 12 & 24 \\
\hline Colitis & -- & 2 & 2 & 1 & -- & - \\
\hline Costado, pleuresía & 59 & 74 & 74 & 51 & 46 & 58 \\
\hline Dentición, comillos, dientes & 14 & 15 & 29 & 41 & 38 & 33 \\
\hline Disentería & 264 & 420 & 402 & 222 & 202 & 293 \\
\hline Empacho & 7 & 6 & 17 & 9 & 9 & 10 \\
\hline Fiebre amarilla & 810 & 21 & 268 & 2 & 1 & 2 \\
\hline Fiebres & 680 & 722 & 772 & 446 & 368 & 387 \\
\hline Gangrena & 9 & 10 & 9 & 4 & 6 & - \\
\hline Mal de 7 días & 2 & 1 & 3 & 2 & 12 & 13 \\
\hline Mal de parto, mal parto, parto & 15 & 14 & 26 & 8 & 28 & 13 \\
\hline Pecho & 5 & 7 & 5 & 1 & 5 & 6 \\
\hline Pulmonía, pulmones & 70 & 68 & 81 & 136 & 150 & 146 \\
\hline Tabardillo & 43 & 39 & 27 & 35 & 27 & 29 \\
\hline Terciana & 7 & 10 & 15 & 1 & 4 & 4 \\
\hline Tisis, tuberculosis, sangre de la boca, tubérculos & 454 & 354 & 280 & 392 & 267 & 359 \\
\hline Tos convulsiva & -- & -- & 1 & 2 & 4 & 3 \\
\hline Ulcerado, úlceras & 22 & 16 & 9 & -- & -- & 11 \\
\hline Viruelas & 2 & 1 & 3 & 88 & 474 & 64 \\
\hline
\end{tabular}

Tomado de Carlota Casalino, La Muerte en Lima en el s. XIX, p. 313 
La Tabla 2 indica que el año más crítico fue 1854 con 810 víctimas de fiebre amarilla, el $13 \%$ del total de muertos de ese año, al siguiente año hubo 21 casos y luego en 1856 hubo 268. Podemos señalar que el año de crisis fue 1854, el de latencia fue 1855 y el de rebrote fue 1856.

\section{ENDEMIA VS. EPIDEMIA}

En la Tabla 3, se puede apreciar que los casos de viruela de los nueve años muestran que en 1840 hubo 3 casos, luego en 1845 fueron 29 , en 1850 se contaron 27 , solo hubo 2 casos en 1854, 1 en 1855, 3 en 1856. Pero en 1858 se eleva a 88, luego en 1859 llegan a ser 474 y en 1860 baja a 66 .

Al comparar el número de víctimas de viruela con el de fiebre amarilla, concluimos que el primero era mucho menor. Probablemente, esto puede deberse a la aplicación de vacunas desde principios de siglo que, si bien no desterró el mal, no produjo las cifras alarmantes del periodo colonial.

Al relacionar las muertes por causas infecciosas con las víctimas de las epidemias, notamos que en "tiempos normales" el porcentaje de muertes por causas infecciosas es alrededor del $70 \%$ y en tiempos de epidemias el porcentaje sube a $75 \%$, llegando en momentos de crisis a ser el $90 \%$ como en 1854 y 1856.

Solamente en 1854, el año más crítico de la fiebre amarilla, las víctimas de epidemia representan el $29,6 \%$ del total de muertes. En el caso de la viruela cuyo año crítico fue 1859 , las víctimas fueron un $22 \%$.

\section{CONDICIONES SANITARIAS EN LIMA}

El hecho que entre las causas de muerte predominen las enfermedades infecciosas como tisis, disentería y "fiebres", ponen de manifiesto una ciudad insalubre.
Estas condiciones se expresaban en agua contaminada, hacinamiento en las viviendas, convivencia con animales de corral y de tiro, inadecuadas instalaciones de agua y desagüe, entre otras. Estamos ante una patología social que afectaba la salud de la población de todos los sectores económicos, siendo los más pobres los más expuestos ${ }^{(8)}$.

En esa época hubo una voz de alerta que hizo evidente esa situación en diversos medios. Manuel A. Fuentes encabezó una campaña para que el Estado se preocupara y tomara medidas a favor de la higiene pública. Con ella se podía prevenir enfermedades y lograr una sociedad mucho más saludable. Fuentes señalaba que: Las que ocasionan [la muerte] para ambos sexos son las fiebres, disentería $y$ tisis ${ }^{(9)}$. A veces se vinculaban los casos de disentería con la falta de higiene en lugares donde se expendían alimentos, como panaderías.

En periodos de epidemias, las autoridades y la población tomaron medidas específicas para afrontarlas. Fue la oportunidad para mejorar las condiciones sanitarias. Problemas como desagües, mantenimiento y limpieza de acequias, canalización de aguas negras, abastecimiento de agua potable, tratamiento y eliminación de la basura, ventilación de los ambientes, alumbrado, hacinamiento, fueron materia de regulación por las autoridades.

El Estado, al incorporar el concepto de higiene pública como parte de sus responsabilidades, invirtió en la mejora de estas condiciones sanitarias, especialmente después de la irrupción de las epidemias, así se tomó conciencia de la importancia de prevenir las enfermedades. Esto se expresó en la legislación municipal de 1860.

\section{CONCLUSIONES}

El número de víctimas por enfermedades endémicas como las "fiebres" o disentería fue más alto que el número de víctimas de epidemias.

Tabla 3. Muertes por causas infecciosas y muertes por epidemias en Lima, periodo 1840 al 1860.

\begin{tabular}{lcccc}
\hline Año & Total muertes & $\begin{array}{c}\text { Total muertes que indi- } \\
\text { can causas }\end{array}$ & $\begin{array}{c}\text { Total muertes por causas } \\
\text { infecciosas }\end{array}$ & $\begin{array}{c}\text { Total de víctimas de fiebre amari- } \\
\text { Ila y/o viruelas }\end{array}$ \\
\hline 1840 & 2339 & 1710 & 1189 & 3 \\
1845 & 3251 & 2463 & 1802 & 29 \\
1850 & 3594 & 2058 & 1493 & 27 \\
1854 & 5949 & 2740 & 2488 & 812 \\
1855 & 4838 & 2299 & 1814 & 22 \\
1856 & 4618 & 2259 & 2052 & 271 \\
1858 & 3377 & 1971 & 1497 & 90 \\
1859 & 3819 & 2158 & 1701 & 475 \\
1860 & 3459 & 2117 & 1499 & 66 \\
\hline
\end{tabular}

Tomado de Carlota Casalino, La Muerte en Lima en el s. XIX, p. 317 
Las endemias se debían a malas condiciones higiénicas de la ciudad dando lugar a una patología social. Las epidemias impactaban más entre la población por su presencia aguda en pocos meses.

La crisis provocada por las epidemias hizo que las autoridades municipales, se preocuparan por mejorar las condiciones sanitarias e impulsaran el concepto de higiene pública como responsabilidad del Estado. Esto se expresó en el reglamento que puso en rigor la municipalidad a partir de 1860.

Además, los cambios y modernización en la ciudad, fueron posible gracias a los inmensos recursos provenientes del guano.

\section{REFERENCIAS BIBLIOGRÁFICAS}

1. Organización Mundial de la Salud. Medidas para contener epidemias presentes y futuras rumbo al año 2030 . MONUUNAM. México, 2016, 11-12. [Citado el 25 de mayo de 2017]. Disponible en: http://www.monuunam.unam. $\mathrm{mx} /$ downloads/HANDBOOK\%20 OMS\%20DEFINITIVO.pdf

2. Salaverry O. Cayetano Heredia (17971861). Rev Peru Med Exp Salud Publica. 2011,28(3) 556-9.

3. Casalino C. La muerte en Lima en el siglo XIX: Una aproximación demográfica, política, social y cultural [Tesis de Maestría]. Lima: Escuela de Posgrado, Pontificia Universidad Católica del Perú; 1999.
4. Patterson C. Amebic Dysentery. En: Kiple Kenneth (ed). The Cambridge World History of Human Disease. USA: Cambridge University Press: 1995 , p. 568 570 y 696 .

5. Mann Ch. C. 1493. Una nueva historia del mundo después de Colón. Madrid: Katz editores; 2013, p. 113-137.

6. Cook N.D. La conquista biológica. Las enfermedades en el Nuevo Mundo. España: Siglo XXI; 2005, p. 107-140.

7. Rottenbacher de Rojas JM. Emociones colectivas, autoritarismo y prejuicio durante una crisis sanitaria: la sociedad limeña frente a la epidemia de fiebre amarilla de 1868 [Tesis de Maestría].
Lima: Escuela de Posgrado, Pontificia Universidad Católica del Perú; 2013.

8. Lossio J. Acequias y gallinazos. Salud ambiental en Lima del siglo XIX. Lima: IEP; 2003.

9. Fuentes MA. Guía Histórico descriptiva administrativa, judicial y de domicilio de Lima 1860-1861. Lima: Librería Central; 1861. Edición fascimilar a cargo de César Coloma; Lima: Instituto Latinoamericano de Cultura y Desarrollo; 1998. p. 55.

Correspondencia: Carlota Casalino

Dirección: Tomás Ramsey 915 - Magdalena

Teléfono: 2624489

Correoelectrónico:pchucase@upc.edu.pe

\section{¡Ahora puede subir su artículo, a través de nuestro sistema Open Journal System (OJS)!}

www.rpmesp.ins.gob.pe

MINISTERIO DE SALUD

Instituto Nacional de Salud
Síguenos en

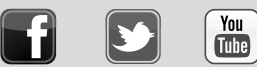

